\title{
Mirror neuron: a beautiful unnecessary concept
}

\begin{abstract}
Mirror neurons theory, which had been put forward in the eighties based on the results of cognitive research experiments on the macaque monkeys, has prima facie been further validated by the extensive cognitive neurosciences investigations of primates and humans, over the past three decades. The concept was initially prompted by the fact that the brain activity patterns of the subjects were nearly similar, whether the activity was performed or observed by them. And presently, learning of various natures and empathy, and perhaps some aspects of survival, are ascribed to the operations of this class of neurons. Obviously the added complexity on the already complex field of neurosciences cannot be underestimated; and of course there are opponents of the theory, and some profound questions have been raised. Present work, though also in opposition, is based on completely different ground: the fact that the ingenious and grand efforts of the proponents of the theory can be explicated in the realm of the established neural structure of the brain and its computational operations. This possibility is based on the recent discovery of the tactile nature of the vision sensation. Ironically all the results, which form the basis of the mirror neuron concept, also serve to provide the conceptual proof of the new vision theory, which preempts any need for the introduction of the new class of neurons. The vision theory, partially validated through the efforts of the development of the tactile vision substitution systems (TVSS) and ironically also by some to the point mirror neuron experimental works, are sufficient to explain the processes behind empathy, learning and perhaps other mental phenomena; and as such, the need for presumption of additional class of neurons is dispelled. The mental phenomena, which rendered the claim of the mirror neurons, are simply the consequence of subjects beings variably touched by the state of the living environment, through the coherent tactile operation of all senses (four already known as having tactile nature); eyes having the most prominent role: It is the brain's response (the computations outputs) as motor cortex activity,-- subsequent to the discernment of the streaming massive tactile input data, to appropriately coordinate the observer's perceived (tactile) engagement, conditioned by the her mental intentional stance sourced in the brain's protocols (acquired neural patterns)-which is misinterpreted as the evidence for the conceptualization of the mirror neuron.
\end{abstract}

Volume II Issue 6 - 202 I

\author{
Jahan N Schad \\ Lawrence Berkeley National Laboratory, USA
}

Correspondence: Jahan N Schad, PhD; Retired LBNL (UCB) Scientist 376 Tharp Drive, Moraga, Ca 94556 Email schadn5@berkeley.edu

Received: September 30, 2021 | Published: December 08, 2021

Keywords: mirror neurons, computational brain, vision, tactile sensation

\section{Background}

The idea of mirror neurons (the dubbing of the name happened later in the research efforts), was promulgated by Dr. Giacomo Rizzolatti. ${ }^{1}$ and his colleague, of the university of Parma, Italy, as a result of their cognition experiments on the macaque monkeys (in 1980s and 1990s), which showed close brain signal patterns, mainly in the motor neuron, when an activity was either performed or observed by the subjects. Such works on primate and humans have been continuing and the article by Ferrari and Rizzolatti, ${ }^{2}$ published in a special issue of the Philosophical Transaction following the workshop held in Ettore Mjorana (Sicily) on the occasion of $20^{\text {th }}$ year of the discovery of the mirror neurons-- provides an account of the past works, and what path and progress that may lie ahead, in their views. Extent of the works on the subject, and the findings, that according to above investigators have "impacted disciplines outside neurosciences, such as psychology, ethology, sociology and philosophy, or that they had interested novelists and laymen," does not allow a deserving review of all the developments of the past 25 years research by the proponents and supports of the mirror neuron theory, and their few opponents, ${ }^{3}$ many are referenced in the Phil. Transaction-especially, considering that they are not of direct relevance to this work, that only benefits from the widely known results of the associated experiments. Nonetheless, the phenomena observed in these experiments, regardless of the contentions of the opponents, are undeniable; but these experimental results on which the mirror neuron theory is built, could be explained in the light of the new theory of vision, ${ }^{4}$ which unifies the nature of vision with those of the other senses. The tactile nature of vision implies observer's participation in what is observed, the consequence of which, explored in this work proves the mirror neuron concept unnecessary.

\section{Synthesis}

The experimental results, and observations, which form the basis of the ingenious "Mirror Neurons Theory", are the direct consequence of brain's discernment of, and response to, the massive streaming tactile signals from the optic nerves. And the logic behind such discernment is put forward in the theory of tactile nature of vision, ${ }^{4}$ which is summarized in the followings:

Prima facie, on the basis of the fact that brain processes tactile afferent signals from four out of five senses, it is not farfetched to imagine that the visual afferent signals, resulting from the matter (modulated) reflected electromagnetic waves' stimulation of millions of retinal nerves, despite being abundant and comparatively more intricate, would be no different in nature than other afferent signals resulting from the matter nerve stimulations of other senses; and be processed, as tactile signals by the brain, albeit through the deployment of more extensive special purpose circuitry, those of the occipital cortex. The data received by the retinal nerves' demodulation (bandpass filtering) of the (matter modulated) EM waves, would similarly contain only matter (tactile) information, to 
eventually render complete and extensive tactile cognition. The works of Bach-y-Rita, and colleagues and others ${ }^{5,6}$ in the development of tactile vision substitution systems (TVSS), prove the tactile nature of vision afferent signals. The TVSS deploys more parts of the brain neural net when massive tactile sensory data becomes available, as in TVSS deployment: Recent studies ${ }^{7}$ of brains segmental activities indicate significant participation of visual cortex in the development of the (tactile) visual sensation in blinds, while it is not the case for the sighted; for the activities of other senses. However, in the TVSS deployment the overall brain neural engagements would expectedly be minuscule compared to that of the natural vision; and that is why TVSS created vision is black and white and mostly sketchy. In TVSS, a vibro-tactile or electro-tactile interface, connected to a system consisting of a camera and processor, is used to pulse stimulate more enervated parts of the skin or tongue coherently, ${ }^{8}$ in order to invoke the central nervous system to create (simulate) a limited vision perception of the field of the camera. A recent National Public Radio broadcast ${ }^{9}$ demonstrated an application of the system. Such experimental feats, demonstrating the success of the tactile vision substitution systems (TVSS) ${ }^{5,6}$ validate (at least partially) the tactile nature of the vision.

Ironically the vision theory benefits further validation by some of the to the point mirror neuron experimental works; ${ }^{10}$ as well as from other comprehensive experimental works aimed at the investigation on the brain substrate activity behind the mirror neuron phenomenon. These include use of TMS (Trans-cranial Magnetic Simulation), ${ }^{11}$ as well as in the fMRI in the works such as those of Keysers et al. ${ }^{10}$ and in Keysers and Gazzola ${ }^{10}$ (e.g. Alaert, et al. ${ }^{12}$ ). Additional support for the tactile vision theory may be found in the work of Aghajan et al., ${ }^{13}$ in which experimental evidence for hippocampal placement activities for both distal visual cues and self-movements is reported.

The tactile nature of vision, summarily established above, provides a basis for a different and correct interpretation of the empathy and other aspects of Qualia that the observers of "touching scenes" report. Crucial point for this claim is in relating the evidence of the specific sites of the brain activity to the known functional operation of the brain. In such context, the observed motor activity is indicative of the output (response) of the computational machinery of the brain to the inputs resulting from the tactile sensing of the vision stimulants. The review work of J.M. Kilner and R.N. Lemon. ${ }^{14}$ bears indisputable evidence to such sites of cortex activity (emphasis added):

"Here we have reviewed what is currently known about mirror neurons in the different cortical areas in which they have been described. There is now evidence that mirror neurons are present throughout the motor system, including ventral and dorsal premotor cortices and primary motor cortex, as well as being present in different regions of the parietal cortex".

Also further example of such depictions is in the following excerpt from the recent work of Carlos E. Vargas-Irwin et al., ${ }^{15}$ which vividly depict brain activity sites in various modes (emphasis added):

"Here we provide direct demonstration that observing, imagining or attempting action activates shared neural ensembles in human motor cortex".

With such evidence of the motor cortex activity in response to watching a scene or an event, the very established facts that underlie the formulation of the mirror neuron theory, find proper explication, which preempts the need for the additional neurons. The empathy mental phenomenon is simply the result of the tactile interaction of the brain with the (eventful) living environment, through the coherent contribution of each of the engaged (stimulated) senses, eyes playing the most prominent role. The discernment of the stimulants, in the form of streaming massive tactile input data, prompts a coordinated brain simulation response (output) in motor signals, which activates the motor cortex. And of course the nature of the response would depend on the (observing) mind's intentional mental stance determined by the brain's existing (learned) patterns; and the presence, or lack thereof, of direct (sparse) bodily involved touch signals. The mirror neuron concept underlying evidences found in brain scans, and other instances, summarized in anecdotal observation "Monkey see, Monkey do," are simply the consequences of physical involvement in the realities of the field of vision; and that watching an event is very much like being physically involved in it, invoking brain response conditioned by the dictates of checks and balances, implemented in the activated motor cortex regions.

Given all the assumptions about the overall engagement of various parts of the neural network of the brain, when called for, -- in addition to the segments which are known to relate to certain functions-- the plasticity property of the brain, and the additional recent claims of the known area of the sensorimotor neuron involved in understanding (all very rational), and given the requirement for the structural multitudinous circuits in artificial neural networks aimed at performing task, as they get more complicated, it is not farfetched to think that in the perception of our (all inclusive) environment, in the context of a unified tactile operation of senses, we are essentially being touched by the world and its events. And that it should not be strange that the brain does not differentiate where the tactile signal comes from; whether our hands form or another sense, which touches us. Expectedly the engagement of the additional senses in performing the task itself would engage more of the neurons of the brain, which explain in some of the reported fMRI brain activity observation; and the results add for better understanding!

\section{Acknowledgments}

None.

\section{Conflicts of interest}

The authors declare no conflicts of interest.

\section{References}

1. Rizzolatti Giacomo, Craighero Laila. "The mirror-neuron system" (PDF). Annual Review of Neuroscience. 2004;27(1):69-192.

2. Ferrari PF, Rizzolatti G. "Mirror neuron research:the past and the future". Philos Trans R Soc Lond B Biol Sci. 2014;369 (1644):20130169.

3. Hickok G. "Eight problems for the mirror neuron theory of action understanding in monkeys and humans". $J$ Cogn Neurosci. 2009;21(7):1229-1243.

4. Schad J.

5. Bach-y-Rita P, Collins CC, Sanders AF, et al. Vision substitution by tactile image projection. Nature. 1968;221:963-964.

6. Bach-y-Rita, Tactile substitution studies. Ann N Y Acad Sci. 2006;1013:8391.

7. AAA public Release. Researchers find the blind use visual brain area to improve other senses, Georgetown University Medical Center (GUMC). 2010 .

8. Bach-y-Rita P. Kaczmarek KA, Tyler ME, et al. Form perception with a 49-point electro-tactile stimulus array on the tongue:a technical note. $J$ Rehabil Res Dev. 1998;35(4):427-430.

9. http://www.radiolab.org/story/painting-tongues/. 
10. Keysers C, Wicker B, Gazzola V, et al. A touching sight SII/PV activation during the observation and experience of touch. Neuron. 2004;42(2):335346.

11. Rizzolatti G, Fogassi L. Mirror neuron mechanism; recent findings and perspective. Phil Trans R Soc. 2014;369(1644):20130420.

12. Alaert K, Swinnen SP, Wendroth N. Is human primary motor cortex activated by muscular or direction-dependent features of observed movements? Cortex. 2009;45:1145-1155.
13. Aghajan ZM, Acharya L, Moore JJ, et al. Impaired spatial selectivity and intact phase precession in two-dimensional virtual reality. Nature Neurosciences. 2015;18:121-125.

14. JM Kilner, RN Lemon. What We Know Currently about Mirror Neurons. Curr Biol. 2013;23(23):R1057-R1062.

15. Vargas-Irwin CE, Feldman JM, King B, et al. Watch, Imagine, Attempt:Motor Cortex Single-Unit Activity Reveals Context-Dependent Movement Encoding in Humans With Tetraplegia. Front Hum Neurosci. 2018;12:450. 